\title{
Research on Explosion Points with the Same Characteristics Matching and Location of Automatic Target-scoring System
}

\author{
Xin Wang ${ }^{\text {a }}$, Xiao Wang, Xuejie Wei and Hongwei Yang \\ North China Institute of Aerospace Engineering, Langfang 065000, China \\ awxtju1987@163.com
}

\begin{abstract}
Keywords: automatic target-scoring system; image matching; same characteristics; epipolar constraint.
\end{abstract}

\begin{abstract}
With the continuous development and intense research, how to solve the problem of remote monitoring and automatic evaluation in salvo bomb mode for the shooting range automatic target evaluation system is one of the issues that need to be addressed. Based on this background, this paper focuses on multi-explosion points in the same characteristics matching and location. Analyzing multi-explosion point's ground situation and the image characteristics in salvo bomb mode, namely technology difficulty, an image processing algorithm based on time division and epipolar constraint is raised
\end{abstract}

\section{Introduction}

This paper is based on the application requirements of multi-explosion points in the same characteristics matching and the distance relative to the bull's-eye with remote monitoring and automatic evaluation in salvo bomb mode. Salvo bomb mode is to point to in a short period of time, generally refers to a few seconds, complete dozens of shell for the launch of the same target, in military sense to strengthen the effect of firefighting. Because of artillery shells firing at the same time in a short time, explosion to the ground in a short period of time, and the same image characteristics, the traditional image matching algorithms do not apply. An image processing algorithm based on time division and epipolar constraint is raised to measurement and display the location of live ammunition explosion points relative to the bull's-eye accurately and timely.

\section{System Architecture}

Multi remote target-scoring system is mainly composed of remote measuring station, level one upper computer and level two monitoring PC three parts. About the two remote measuring station on left and right and an upper computer through McWiLL network compose of a set of independent target-scoring system, as shown in figure 1.

Remote measuring station includes high resolution measurement sensor unit (gigabit network cameras), computers and McWiLL manpack terminal. Upper computer display training record real-time for lists, histogram and explosion points schematic diagram ${ }^{[1]}$. When monitoring multiple target area at the same time, the level two monitoring PC gains each PC synchronous measurement data, and to centralized control and display them through the RS - 485 bus.

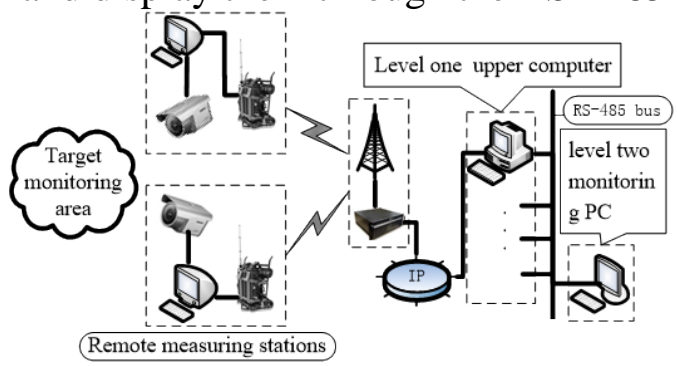

Figure 1. A set of target-scoring system in Multi remote target-scoring system schematic diagram 


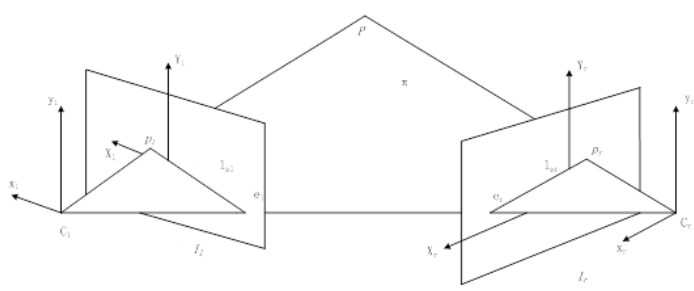

Figure 2. Epipolar relations in binocular stereo vision measurement

\section{Same Image Points Matching based on Epipolar Constraint}

As shown in Figure 2, represents epipolar geometry in a binocular stereo vision measuring system. In the figure, $I_{l}, I_{r}$ are respectively the left and right camera image planes, $C_{l}, C_{r}$ are respectively the left and right optical centers of two cameras, $p_{l}, p_{r}$ are the projection points in the image planes both left and right a point $\mathrm{P}$ in the space. $\overline{C_{l} C_{r}}$ is a baseline which connects two optical centers. $\mathrm{P}$ point and two cameras optical center $C_{l}, C_{r}$ determine the polar plane $\pi$. The intersection $e_{l}, e_{r}$ of the baseline and the image planes of the two cameras are the poles. The intersecting lines $\overline{e_{l} p_{l}}, \overline{e_{r} p_{r}}$ are pole lines two camera image planes with the polar plane ${ }^{[2]}$.

In the figure, the straight line $\overline{e_{l} p_{l}}$ is the pole line the left camera image plane corresponding to the point $p_{r}$, the straight line $\overline{e_{r} p_{r}}$ is the pole line the right camera image plane corresponding to the point $p_{l}$. From epipolar constraints, as the projection points for the same point $\mathrm{P}$ in space, the point $p_{r}$ corresponding to the point $p_{l}$ must lie within the right camera image plane pole line $\overline{e_{r} p_{r}}$, the point $p_{l}$ corresponding to the point $p_{r}$ must lie within the left camera image plane pole line $\overline{e_{l} p_{l}}$. Epipolar constraints is the corresponding point and line, the search matching space which the points corresponding to is compressed from the whole image to only in the vicinity of the pole line, or within the line, which is a very important role for the matching points.

Two video camera projection equations (1):

$\left\{\begin{array}{l}s_{l} p_{l}=M_{l} X_{w}=\left(M_{l 1}, m_{l}\right) X_{w} \\ s_{r} p_{r}=M_{r} X_{w}=\left(M_{r 1}, m_{r}\right) X_{w}\end{array}\right.$

In the formula, $p_{l}, p_{r}$ are respectively homogeneous spatial coordinates of the image point $\mathrm{P}$ in the left and right two cameras, $M_{l}, M_{r}$ are respectively the projection matrix of the left and right cameras, $X_{w}$ is the point $\mathrm{P}$ homogeneous spatial coordinates in the world coordinate system. $M_{l}, M_{r}$ are the $3 \times 4$ matrix, $M_{l 1}, M_{r 1}$ are to the left $3 \times 3$ parts respectively, $m_{l}, m_{r}$ show that the last column on the right respectively, $X_{w}$ is represented as $X_{w}=\left(X^{T}, 1\right)^{T}, X=\left(X_{w}, Y_{w}, Z_{w}\right)^{T}$, where it is converted to the formula (2):

$\left\{\begin{array}{l}s_{l} p_{l}=M_{l 1} X+m_{l} \\ s_{r} p_{r}=M_{r 1} X+m_{r}\end{array}\right.$

Remove $X$ :

$s_{r} p_{r}-s_{l} M_{r 1} M_{l 1}^{-1} p_{l}=m_{r}-M_{r 1} M_{l 1}^{-1} m_{l}$

After conversion available:

$p_{r}^{T} F p_{l}=0$

$F=[m]_{\times} M_{r 1} M_{l 1}^{-1}$ is the basic matrix.

Decompose formula according to the analytical form of the fundamental matrix:

$l_{p_{r}}=F p_{l}$ 


$$
l_{p_{l}}=F^{T} p_{r}
$$

the formula (5) (6) are respectively pole lines that the left camera image points corresponding to the right camera image and the right camera image points corresponding to the left camera image.

After parsing the above, it is clear that based on how to determine the location of the left and right pole lines in known point camera image, greatly reducing the scope of the search to find the corresponding point, providing a basis to points matching in the same name, reducing the search complexity.

\section{Technical Difficulties of Multi-explosion Points in the Same Characteristics Matching and Location}

System design in front of the camera equipped with $760 \mathrm{~nm}$ infrared filter. The explosion strong light is as image acquisition signal. Explosion point's image in salvo bomb mode is more time sequence continuous distribution characteristics ${ }^{[3]}$.In consideration of the system adopts industrial camera is 20 frames per second, even if the time is very short, but considering the camera acquisition speed, explosion points will continue to appear in the image on the different frames. So that it can use multiple explosion points on the time series of distribution to complete points matching. At the same time, completely synchronization of the camera image frames is difficult. There are multiple explosion points in the same frame image. Explosion points has the same characteristics consistent.

Through the analysis found that salvo bomb mode due to the different operational mechanism, bombs in a different way, contrast injective bombing mode has its own characteristics. Combined with the working principle of the system, it gets the problem of the technical difficulties above need to solve. These problems also need to be consider in the process of algorithm design.

\section{Explosion Points Matching and Locating Algorithm}

Remote Measuring Station Algorithm of Image Processing. Remote measuring station mainly complete image acquisition, explosion points automatic detection and recognition process. To collect all explosion points in salvo bomb mode , and avoid explosion points information is missing, fully consider the actual application, using real time interframe difference threshold judgment triggers to start, acquiring fixed enough frames image. Then using connected domain statistics preliminary detect of explosion points. Appeared to rule out the same explosion point on multiple consecutive frames to recognize by mistake, repeated recognition, this paper proposes a method of sequence image spatial and temporal correlation detection-continuous frame judgment. According to the explosion points region centroid and the external rectangular frame on rest frames corresponding position to decide. After the remote measuring station algorithm of image processing, it can get multiple explosion points frame number, centroid image plane coordinates, the explosion point area pixel size and other information in chronological order.

After the remote measuring station algorithm of image processing, it gets real explosion point area in the frame number. As shown in figure 3 to 4, figure 3 relative to a frame image live ammunition, figure 4 to use the remote measuring station algorithm of image processing, there are four explosion points block. Multiple explosion points frame number, centroid image plane coordinates, the explosion point area pixel size are uploaded to the upper computer through McWill wireless networks.

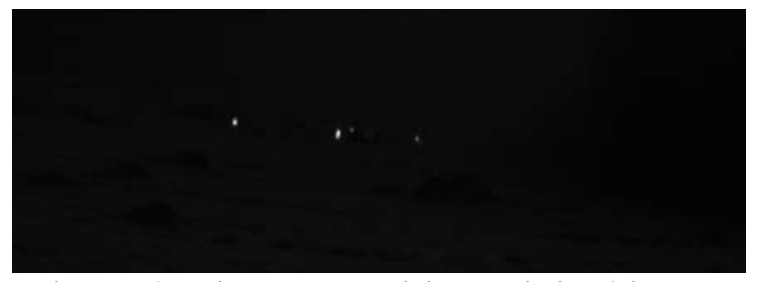

Figure 3. Live ammunition original image 


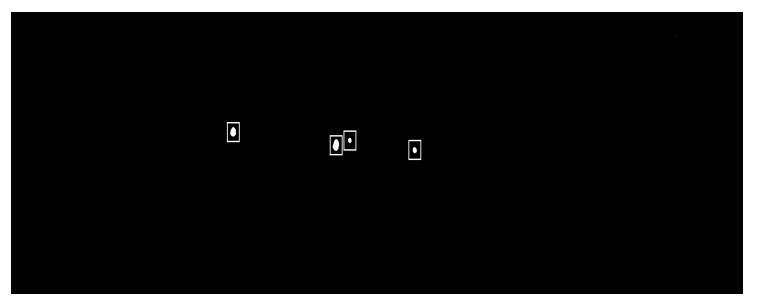

Figure 4. Image after the remote measuring station algorithm of image processing

Upper Computer Algorithm of Image Processing. Upper computer is mainly responsible for explosion point matching using the information that two remote measuring stations upload. Using time series segmentation matching algorithm, explosion points are divided and in statistics again from the angle of time distribution. Explosion points are into multiple frame periods, so that the explosion points matching range narrowed to a frame section, decompose the complex problems, avoid all the explosion points match together. Within each frame period, using polar constraints, identifying the corresponding explosion points.

Based on the polar geometric relationships and polar constraint conditions of binocular stereo vision measurement ${ }^{[4]}$, the following two outfield incandescent light bulb simulation experiments verify the feasibility of matching based on polar constraint conditions.

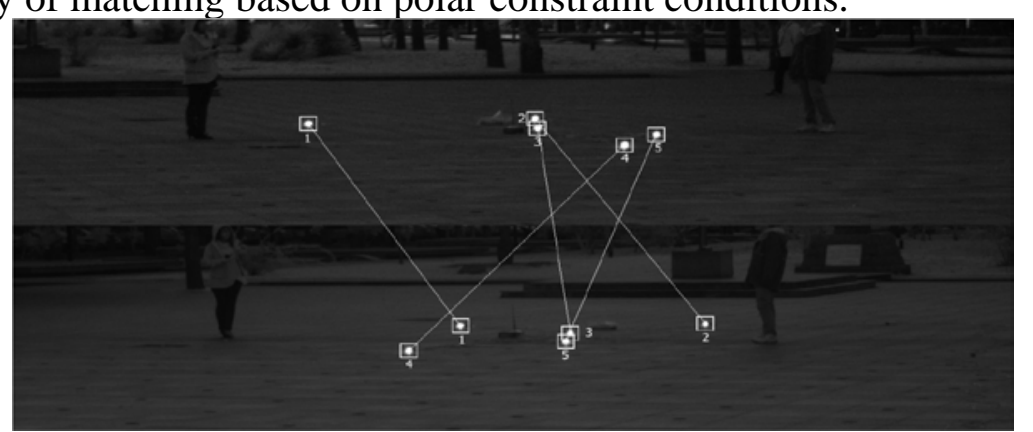

Figure 5. The experiment image of five incandescent light bulbs

Five incandescent light bulbs are put any. Figure 5 is the experiment image, incandescent light bulbs are marked by Arabic Numbers on the figure with the same number.

\section{Summary}

Explosion points matching and locating algorithm is proposed including five algorithm modules. Especially time division and epipolar constraint algorithm modules to solve the characteristics consistent multi-objects matching and positioning problem have certain effect.

\section{Acknowledgment}

The research is supported by North China Institute of Aerospace Engineering Youth Fund Project (NO.KY-2013-13), the Science and Technology Research and Development Project of Langfang City (NO.2014011023).Corresponding author, Xin Wang,email,wxtju1987@163.com.

\section{References}

[1] Xu Jiayun. Moving target detection and tracking in image sequence method research. Nanjing: nanjing university of science and technology, 2008.

[2] Zhang Guangjun, Vision Measurment, Beijing: Science Press2008, 145-147.

[3] Han Shuanglai, Range impact coordinate measuring system research of some key problems, tianjin: tianjin university, 2006

[4] Zhang Guangjun, Visual measurement, Beijing: science press, 2008145-147 